\title{
Benefits of Clipping Surgery Based on Three-Dimensional Computed Tomography Angiography
}

\author{
Mutsumi NAGAI and Eiju WATANABE* \\ Department of Neurosurgery, Sano Kousei General Hospital, Sano, Tochigi; \\ *Department of Neurosurgery, Jichi Medical School, Shimotsuke, Tochigi
}

\begin{abstract}
The recent development of three-dimensional computed tomography (3D-CT) angiography with multi detector row CT (MDCT) improves cerebral artery imaging due to faster scanning at thinner collimation over a large scanning volume. Aneurysm clipping was performed using only $3 \mathrm{D}-\mathrm{CT}$ angiography created by MDCT with 16 detector arrays (16-MDCT angiography), in place of digital subtraction angiography (DSA) to evaluate the suitability of 16-MDCT angiography for preoperative examination before aneurysm clipping, and identify the essential points when making 3D images. Thirty-two patients with subarachnoid hemorrhage (SAH) identified by initial CT or with non-ruptured aneurysm were entered in this study. Twenty-six SAH patients underwent 3D-CT angiography, and aneurysms were treated by clipping $(n=25)$ or coil embolization $(n=4)$. One patient with no aneurysm depicted by 3D-CT angiography underwent conventional DSA twice subsequently. The sensitivity of 16-MDCT angiography to depict aneurysms in patients with $\mathrm{SAH}$, and time between admission and entering the operating room were evaluated, and compared with those under the former protocol using DSA. The sensitivity of 16-MDCT angiography for aneurysm detection was $100 \%$. Shapes of all aneurysms depicted by 3D-CT angiography were validated intra-operatively. The mean operation waiting time was 2.8 hours, with the shortest time being 1.5 hours. Re-bleeding occurred in one of the 20 patients. We conclude that shorter preoperative waiting time decreased the incidence of premature re-bleeding, resulting in better outcomes. We highly recommend that the surgeon sets the threshold value, and rotates the 3D image to establish better surgical orientation.
\end{abstract}

Key words: three-dimensional computed tomography angiography, cerebral aneurysm, clipping, multi detector row computed tomography, image

\section{Introduction}

Subarachnoid hemorrhage (SAH) caused by intracranial aneurysm rupture is a critical illness, particularly when aneurysm re-bleeding occurs, resulting in a poor prognosis. Intra-arterial digital subtraction angiography (DSA) has long been considered the "gold standard" diagnostic method for identifying an aneurysm. However, DSA causes an enormous burden and discomfort for the patients due to the long examination time, which may increase the risk of re-bleeding, especially within 6 hours after SAH onset. ${ }^{18)}$ In addition, DSA is invasive and the complication rate of neurological deficit is between $0.25 \%$ and $1 \%$. Therefore, the time from admission to clipping surgery must be shortened to decrease the risk of presurgical re-bleeding. Three-dimen-

Received November 19, 2009; Accepted March 3, 2010 sional computed tomography (3D-CT) angiography has various advantages as a diagnostic tool for presurgical examination. ${ }^{2,3,6,7,10,12,15-17,20,22,23,25,26,29-32 \text {, }}$ 34,36-39) Some authors claim aneurysm clipping can be done only with 3D-CT angiography information. ${ }^{5,14,16,24,27,33,40)}$ However, whether 3D-CT angiography offers sufficient presurgical information for all cerebral aneurysms remains controversial.

Development of 3D-CT angiography based on multi detector row CT (MDCT) has led to improved cerebral artery imaging due to faster scanning with thinner collimation over a larger scanning volume. Since 2003, our institute has used MDCT angiography with 16 detector arrays (16-MDCT angiography) to obtain detailed anatomical information about cerebral aneurysms and the surrounding vessels. Therefore, cerebral aneurysms have been treated since November 2003 using only 16-MDCT angiography in a diagnostic and preoperative planning study. 
In many reports, the clipping surgeon did not always create the 3D-CT angiography images, as often a radiologist and/or technician operated the 3D-CT angiography workstation and created the 3D images. In our institute, the 3D images are created by the clipping surgeon to clearly image the aneurysm, the surrounding arteries, and the adjacent bony structures. Therefore, we assume that 16-MDCT angiography allows the surgeon to perform clipping safely with clear planning based on only 3D-CT angiography information.

The present study investigated the diagnostic information and quality of 3D-CT angiography for preoperative planning. The waiting time and rate of preoperative re-bleeding were also assessed. The technique and essential procedures to obtain adequate preoperative information using $3 \mathrm{D}-\mathrm{CT}$ angiography are also described.

\section{Materials and Methods}

When a diagnosis of SAH was established by CT on admission, 3D-CT angiography images were obtained using 16-MDCT angiography. If the 3D-CT angiography showed aneurysms in the anterior circulation, such as the internal carotid artery (ICA), anterior communicating artery (AcomA), anterior cerebral artery, and middle cerebral artery (MCA), aneurysm clipping was performed based only on the 3D-CT angiography data. If 3D-CT angiography did not show any aneurysm, conventional DSA was subsequently performed. If the ruptured aneurysm was located in the posterior circulation, the patient was transferred for interventional coil embolization.

A total of 32 patients with SAH (excluding traumatic SAH cases) or non-ruptured aneurysm were admitted to our institute from 2004 to 2006. Three patients did not undergo clipping surgery or coil embolization because they were deeply comatose or elderly. One of these three patients did not undergo 3D-CT angiography and one patient had SAH of unknown origin diagnosed by repeated DSA following 3D-CT angiography. Therefore, 25 aneurysms were treated by craniotomy, and 4 were treated using endovascular coil embolization. Five of the 25 aneurysms treated surgically were non-ruptured. Age, location of the aneurysm, clinical grade according to the Hunt and Kosnik scale, amount of subarachnoidal blood according to the Fisher classification scheme, and Glasgow Outcome Scale were evaluated. The time from admission to surgery was calculated for patients with SAH who were treated by emergency aneurysm clipping surgery.

3D-CT angiography was performed as follows. Regions of interest (ROIs) for bolus tracking were set in the right common carotid artery. All scans were initiated manually (about 3 seconds) after once the technician confirmed ROIs with the contrast medium. The mean scanning delay was 27 seconds. Before the contrast medium was administered, saline injections were administered with the patient's arm in the scanning position to ensure successful vein cannulation. Next, $50 \mathrm{ml}$ of contrast medium (Omnipaque 300 syringe; Daiichi Sankyo Co., Ltd., Tokyo) was administered intravenously at a flow rate of 3.5 $\mathrm{ml} / \mathrm{sec}$. After the contrast medium was administered, $50 \mathrm{ml}$ of saline was injected using a double-syringe power injector (Dual Shot; Nemoto Kyorindo Co., Ltd., Tokyo). Scanning was performed with an MDCT scanner (Aquillion; Toshiba Corp., Tokyo) with 16 detector arrays. The scans were obtained with a detector configuration of 0.5 $\mathrm{mm}$, pitch of 11 (high speed mode), reconstruction instrument of $0.5 \mathrm{~mm}$, and slice thickness of $0.4 \mathrm{~mm}$. The gantry rotation time was 0.5 seconds. All scanning was done in the direction of caudal to cranial from the basion to squamous suture. The mean scanning time was 7.6 seconds and the mean scanning range was $70 \mathrm{~mm}$. X-ray tube voltage of $120 \mathrm{kV}$ and mean current of $200 \mathrm{~mA}$ were used in all examinations.

All CT scans were transferred to a workstation (M900 Quadra; Ziosoft, Inc., Tokyo). When the 3DCT angiography data were reconstructed, the clipping surgeon manipulated the workstation and created the 3D-CT angiography images. First, the threshold value was adjusted to just below artifactual noise, which blunted the anatomical structure of the arteries such that the aneurysm and surrounding arteries were visible. Then, the 3D images were rotated to reveal the surgical view depicting the $3 \mathrm{D}$ relationships around the aneurysm neck referred for clipping. If the aneurysm was located at the MCA, more views from several directions were added because MCA aneurysms can rotate due to the brain retraction caused by the spatula. If necessary, images were created with the sphenoid ridge partially removed to simulate a more accurate surgical view.

To prevent delayed ischemic neurological deficit, fasudil hydrochloride $(90 \mathrm{mg}$ ) was administered every day from 2 to 14 days after the operation to all patients.

\section{Results}

3D-CT angiography showed no aneurysm in one of the 26 patients with SAH. DSA showed no aneurysm despite being repeated twice. The remaining 25 patients had aneurysms diagnosed with 3D-CT angiography and confirmed during surgery. The 
aneurysm size ranged from 1.08 to $23 \mathrm{~mm}$ in diameter. Therefore, the sensitivity of $3 \mathrm{D}-\mathrm{CT}$ angiography for aneurysm detection was $100 \%$ (false negative $0 \%$ ).

To examine whether the 3D-CT angiography method was quicker than the conventional DSA method, time spent from hospital admission to the operating room was evaluated. We excluded cases in which the operation was intentionally delayed, such as when the patient's condition was too severe for an emergent operation, the surgeon could not participate in the treatment at hand, the patient was admitted to our hospital later than the day of SAH onset, or the aneurysm was located in the posterior circulation. The mean preoperative time was 2.8 hours (range 1.5 to 4 hours). The mean time required for the conventional DSA examination (data calculated from 12 cases prior to 2003) was 4.1 hours.

One patient with right MCA aneurysm showed rebleeding 45 minutes after admission, and became comatose. All other patients maintained consciousness and their general condition remained stable before operation. Patient characteristics and outcomes are listed in Table 1 . Of the 25 patients who underwent clipping, one patient died of extensive cerebral infarction during a period of vasospasm and pneumonia, 21 patients had a good recovery, 1 patient became moderately disabled, and 2 patients became severely disabled.

Representative Case 12: A 52-year-old man visited our institute because of sudden loss of consciousness. CT showed diffuse SAH, thicker in the left sylvian fissure (Fig. 1A). 3D-CT angiography showed an aneurysm at the left MCA bifurcation with a diameter of $10 \mathrm{~mm}$ (Fig. 1B, C). Based on the CT angiography finding, the patient underwent aneurysm clipping surgery 5 hours after admission without additional DSA (Fig. 1D). After surgery he was discharged without neurological deficits.

Representative Case 24: A 71-year-old woman was referred to our institute with a non-ruptured right MCA aneurysm, incidentally found on a routine magnetic resonance imaging checkup. 3D-CT angiography was then performed. In the process, a part of the bony structures around the ipsilateral orbit was digitally deleted to visualize the aneurysm in

Table 1 Patient characteristics

\begin{tabular}{|c|c|c|c|c|c|c|c|}
\hline Case No. & Age (yrs) & Aneurysm rupture & Aneurysm location & $\mathrm{H} \& \mathrm{~K}$ grade & Fisher group & Time to operation (hrs) & GOS \\
\hline 1 & 75 & yes & rt IC-PC & 1 & 3 & 2.5 & GR \\
\hline 2 & 46 & yes & AcomA & 2 & 2 & 2.5 & GR \\
\hline 3 & 76 & yes & AcomA & 1 & 3 & 19 & $\mathrm{D}$ \\
\hline 4 & 56 & yes & lt MCA & 1 & 2 & 4 & GR \\
\hline 5 & 72 & yes & rt MCA & 1 & 3 & 3 & GR \\
\hline 6 & 63 & yes & lt IC-PC & 2 & 2 & 3 & GR \\
\hline 7 & 70 & yes & rt IC-PC & 1 & 3 & 3 & GR \\
\hline 8 & 78 & yes & lt IC-PC & 3 & 3 & 2 & GR \\
\hline 9 & 55 & yes & AcomA & 1 & 2 & 2.5 & GR \\
\hline 10 & 52 & yes & rt MCA & 3 & 2 & 3 & GR \\
\hline 11 & 55 & yes & rt MCA & 2 & 3 & 4 & GR \\
\hline 12 & 52 & yes & lt MCA & 3 & 3 & 5 & GR \\
\hline 13 & 43 & yes & rt IC-PC & 2 & 2 & 6.5 & GR \\
\hline 14 & 48 & yes & AcomA & 2 & 2 & 3 & GR \\
\hline 15 & 65 & yes & rt MCA & 3 & 3 & 3.5 & MD \\
\hline 16 & 55 & yes & lt IC-PC & 2 & 2 & intentionally delayed & GR \\
\hline 17 & 43 & yes & AcomA & 5 & 3 & 1.5 & SD \\
\hline 18 & 70 & yes & $\mathrm{A}_{2}$, lt MCA & 2 & 2 & intentionally delayed & GR \\
\hline 19 & 70 & yes & rt IC-PC & 4 & 3 & 11 & GR \\
\hline 20 & 57 & yes & rt MCA & 5 & 4 & 2.5 & SD \\
\hline 21 & 66 & no & rt IC-PC & 0 & 1 & N/A & GR \\
\hline 22 & 59 & no & rt IC-PC & 0 & 1 & N/A & GR \\
\hline 23 & 23 & no & rt MCA & $1 \mathrm{a}$ & 1 & N/A & GR \\
\hline 24 & 71 & no & rt MCA & 0 & 1 & N/A & GR \\
\hline 25 & 53 & no & MCA & 0 & 1 & N/A & GR \\
\hline
\end{tabular}

AcomA: anterior communicating artery, D: dead, GOS: Glasgow Outcome Scale, GR: good recovery, H\&K: Hunt and Kosnik, IC-PC: internal carotid-posterior communicating artery, MCA: middle cerebral artery, MD: moderately disabled, N/A: not available, SD: severely disabled. 

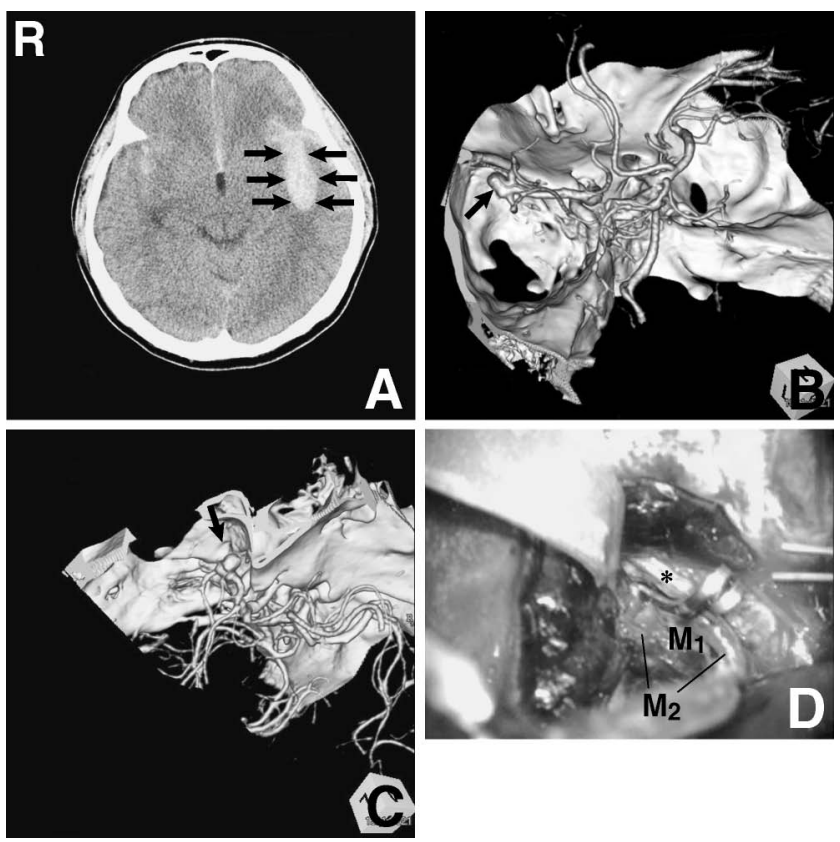

Fig. 1 Case 12. A: Computed tomography (CT) scan demonstrating subarachnoid hemorrhage with thick hematoma at the left sylvian fissure (arrows). B: Threedimensional CT (3D-CT) angiogram showing an aneurysm (arrow) of $1 \mathrm{~cm}$ diameter at the left middle cerebral artery bifurcation. C: 3D-CT angiogram simulating the operative view showing the aneurysm (arrow). D: Intra-operative photograph showing the aneurysm (asterisk), left $M_{1}$ portion, and $M_{2}$ portions.

the "surgeon's view angle" (Fig. 2A-C). The 3D image was evaluated during surgery and compared with the actual aneurysm and surrounding vessel branches (Fig. 2D).

In three of nine internal carotid-posterior communicating artery aneurysms, the anterior choroidal arteries were well visualized by 3D-CT angiography. 3D-CT angiography revealed the anterior choroidal artery in one case with standard manipulation (Fig. $3 \mathrm{~A}$ ) since the artery was thick enough to be depicted in the 3D image. Image accuracy was confirmed during surgery (Fig. 3B). In the other two cases, careful control of the threshold level was necessary to better visualize the anterior choroidal arteries (Fig. 4).

\section{Discussion}

3D-CT angiography has advanced in recent years, particularly with MDCT angiography with spatial resolution almost equal to conventional cerebral DSA. On the other hand, the common MDCT method has a limit to depict an aneurysm because of the slice thickness. Therefore, the resolution of
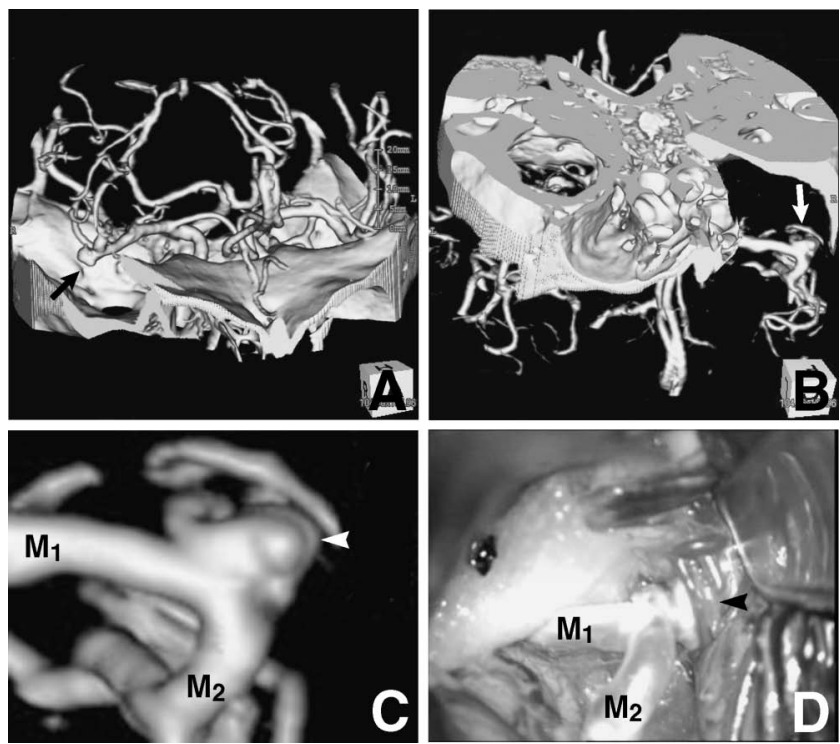

Fig. 2 Case 24. A: Three-dimensional computed tomography (3D-CT) angiogram showing an unruptured aneurysm with small blebs located at right $M_{1}-M_{2}$ junction (arrow). B: 3D-CT angiogram simulating the operative view, with the image is tilted in the direction of the vertex down. The right sphenoid ridge and the orbital roof are digitally cut off (arrow). C: Higher magnification of the 3D-CT angiogram showing the right middle cerebral artery (MCA) aneurysm with blebs (arrowhead), and the $M_{1}$ and $M_{2}$ portions. D: Intra-operative photograph showing the aneurysm (arrowhead), and $M_{1}$ and $M_{2}$ portions of the right MCA. Intra-operative view is quite similar to the 3D-CT angiogram (C).
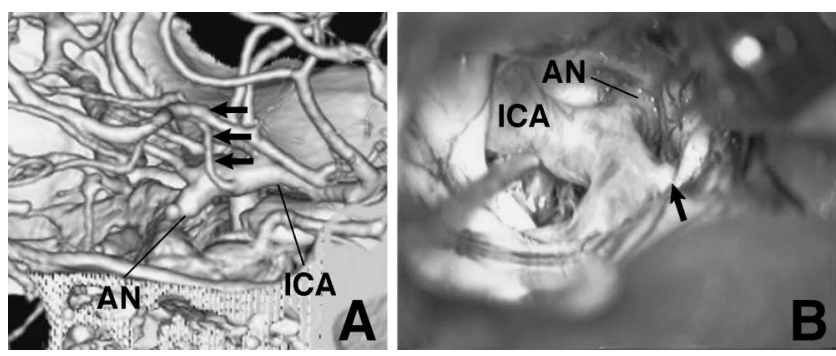

Fig. 3 Case 22. A: Three-dimensional computed tomography (3D-CT) angiogram revealing a right internal carotid artery (ICA), an internal carotid-posterior communicating artery aneurysm (AN), and the right anterior choroidal artery (arrows). B: Intra-operative photograph showing the relatively thick anterior choroidal artery (arrow), the right ICA, and the AN.

MDCT is still below that of conventional DSA. However, following the protocol that patients with no aneurysm on 3D-CT angiography should undergo DSA, all aneurysms would be detected. The sensitiv- 

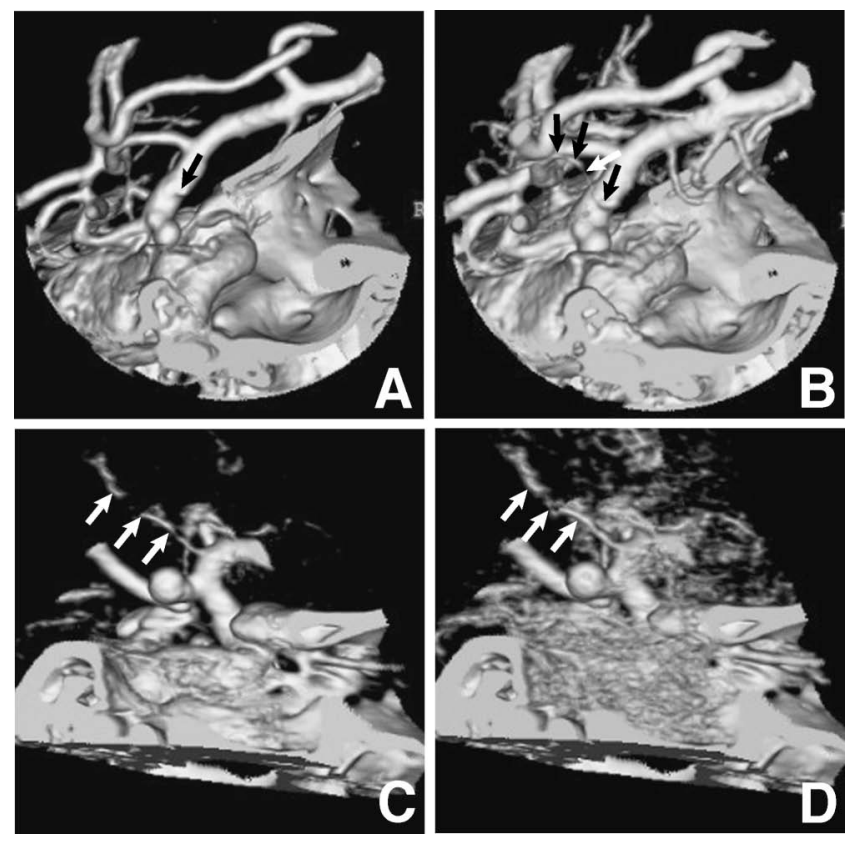

Fig. 4 Three-dimensional computed tomography (3DCT) angiograms illustrating the process of improved visualization of the anterior choroidal arteries by lowering the threshold to a level with good signal-to-noise ratio. A, B: 3D-CT angiograms showing two images of a right internal carotid-posterior communicating artery aneurysm. With the threshold set higher (A), the aneurysm and infundibular base of the anterior choroidal artery (arrow) are visible, but visualization of the anterior choroidal artery is insufficient. With the threshold set lower (B), the anterior choroidal artery is well depicted (arrows). C, D: 3D-CT angiograms of another case. With the threshold set higher (C), the anterior choroidal artery is depicted, but disconnected (arrows). With the threshold set lower (D), the anterior choroidal artery is continuously depicted (arrows), but the image is noisy.

ity for detection of ruptured aneurysm was $100 \%$ ( $0 \%$ false negative) in our study, showing that for an anterior circulation aneurysm, only 3D-CT angiography is required before surgery. Recently, a study showed the efficacy of MDCT for detection of aneurysms, with $0.1 \%$ false negative rate of $3 \mathrm{D}-\mathrm{CT}$ angiography based on subsequent DSA. Although the MDCT used in the study had 64 detector arrays, the slice thickness was $0.5 \mathrm{~mm}$, almost the same as our MDCT. Therefore, 3D-CT angiography can be performed at first to detect aneurysm in the $\mathrm{SAH}$ treatment protocol and only patients with no apparent aneurysm need undergo subsequent DSA.1) However, the false negative rate was different from our data $(0 \%)$, so we expect to find false negatives with an increase in the number of cases. Finally, conventional DSA is still needed to detect all un- detected aneurysms after 3D-CT angiography. However, we think that the information provided by 3D-CT angiography is enough to perform clipping of any detected aneurysm.

About $60 \%$ of aneurysm re-bleeding occurs within 6 hours after the first SAH. ${ }^{18)}$ Moreover, DSA within 6 hours after SAH is significantly associated with a high incidence of re-bleeding. ${ }^{4,18,19)}$ The risk of rebleeding increased from $2 \%$ to $5 \%$ when conventional DSA was performed, and several other reports found DSA increased the risk of re-bleeding. ${ }^{4,18,21,28)}$ Unfortunately, when re-bleeding occurred during DSA, the outcome worsened..$^{8,13,21,28,35)}$ Therefore, performing clipping surgery as early as possible without angiography is preferable. In our protocol, the mean time after patient admission until entering the operating room was 2.8 hours, which is significantly shorter than our previous protocol using angiography (4.1 hours). Our series included only one case of re-bleeding after adopting the new protocol, suggesting that this shorter waiting time lowered the incidence of re-bleeding, and so improved overall treatment outcome.

Accurate 3D images, including skull base images, are essential. 3D images with bony structures provide some benefits. ${ }^{3,5,9,11,14,24,27,33)}$ Imaging the location with respect to the skull base is very useful before surgery, specifically the height of the aneurysm when located at the ICA, the relationship between the sphenoid wing and the aneurysm when located at the MCA, and the relationship between the optic canal and the aneurysm when located at the AcomA. These are all very important points for clipping surgery and are more available in 3D images than bonesubtracted DSA images. The subtraction technique is not necessary unless the aneurysm is located distal to the $\mathrm{C}_{3}$ portion of the ICA. Two types of visualization are used when making 3D-CT angiography images to plan a pterional approach; the first includes the whole skull base, and the second cut off the sphenoid ridge on the operative side. The orbital roof is also cut out if it obstructs image viewing on the computer. Rotation of the $3 \mathrm{D}$ image is also important to simulate the surgeon's view. If the aneurysm is located at the ICA, the parent artery will not move intra-operatively. Thus, the preoperative $3 \mathrm{D}$ images are almost identical to the operative view. However, if the aneurysm is located at the MCA, the slightly twisted parent artery will move intra-operatively as the surrounding arteries are displaced with the motion of the frontal or temporal lobe, dislocated by the spatula. However, we found little discrepancy between the operative view and the estimated 3D images when clipping MCA aneurysm. Therefore, if a more realistic operative 
view of the MCA aneurysm is required, images should be constructed from a deeply tilted angle than estimated. To obtain an absolute intra-operative image, preparation of several images with several different view angles besides that estimated is recommended.

If the surgeon plans to treat ICA aneurysms, recognition of the surrounding arteries (anterior choroidal artery and posterior communicating artery) is important. Most of the posterior communicating arteries are visible on 3D-CT angiography by standard manipulations, but the thin anterior choroidal artery is hard to view. The surgeon has to set the threshold lower to identify the artery at the expense of noise. Even though the images showing the anterior choroidal artery were very noisy, the surgeon could still relate the artery and aneurysm in 3D prior to the operation.

The most essential part of this procedure is that the 3D-CT angiography images should be made by the surgeon who is performing the operation. Rotating 3D images and setting the threshold value on the computer gives the surgeon important information and creates remarkable intra-operative orientation. The imaging time requires less than 20 minutes, which is compatible to the time for hemostasis after angiography. In addition, if the surgeon performs hemostasis manually, tiring of the hand would interfere with fine manipulations of any additional surgery.

3D-CT angiography has a few disadvantages compared to DSA. Until recently, 3D-CT angiography required injection of a single bolus of $80-100 \mathrm{ml}$ of contrast medium ( $300 \mathrm{mg} / \mathrm{ml})$, which is larger than that used for conventional DSA. This is problematic in patients with renal dysfunction. Such a large amount also inhibits repeating the procedures if motion artifacts obscure the images. In an effort to reduce the amount of contrast medium for 3D-CT angiography, we try to inject $50 \mathrm{ml}$ immediately followed by a flash $50 \mathrm{ml}$ of normal saline. This enables us to obtain $3 \mathrm{D}$ images with half of the usual medium, allowing repetition of 3D-CT angiography even in patients with renal dysfunction who could tolerate the examination with this smaller amount of contrast medium.

We conclude that this updated multi-slice CT method will allow us to perform clipping of the identified aneurysm, and that the clipping surgeon should participate in creating the 3D-CT angiography images of the patient. 3D-CT angiography is very useful not only for aneurysm detection but also for preoperative planning.

\section{References}

1) Agid R, Andersson T, Almqvist H, Willinsky RA, Lee SK, terBrugge KG, Farb RI, Soderman M: Negative CT angiography findings in patients with spontaneous subarachnoid hemorrhage: when is digital subtraction angiography still needed? AJNR Am J Neuroradiol 31: 696-705, 2010

2) Alberico RA, Patel M, Casey S, Jacobs B, Maguire W, Decker R: Evaluation of the circle of Willis with three-dimensional CT angiography in patients with suspected intracranial aneurysms. AJNR Am J Neuroradiol 16: 1571-1578, 1995

3) Anderson GB, Steinke DE, Petruk KC, Ashforth R, Findlay JM: Computed tomographic angiography versus digital subtraction angiography for the diagnosis and early treatment of ruptured intracranial aneurysms. Neurosurgery 45: 1315-1320, 1999

4) Aoyagi N, Hayakawa I: Rerupture of intracranial aneurysms during angiography. Acta Neurochir (Wien) 98: 141-147, 1989

5) Boet R, Poon WS, Lam JM, Yu SC: The surgical treatment of intracranial aneurysms based on computer tomographic angiography alone-streamlining the acute management of symptomatic aneurysms. Acta Neurochir (Wien) 145: 101-105, 2003

6) Chappell ET, Moure FC Good MC: Comparison of computed tomographic angiography with digital subtraction angiography in the diagnosis of cerebral aneurysms: a meta-analysis. Neurosurgery 52: 624-631, 2003

7) Dorsch NW, Young N, Kingston RJ, Compton JS: Early experience with spiral CT in the diagnosis of intracranial aneurysms. Neurosurgery 36: 230-236, 1995

8) Earnest F 4th, Forbes G, Sandok BA, Piepgras DG, Faust RJ, Ilstrup DM, Arndt LJ: Complications of cerebral angiography: prospective assessment of risk. AJR Am J Roentgenol 142: 247-253, 1984

9) Futami K, Nakada M, Iwato M, Kita D, Miyamori T, Yamashita J: Simulation of clipping position for cerebral aneurysms using three-dimensional computed tomography angiography. Neurol Med Chir (Tokyo) 44: 6-12, 2004

10) Harbaugh RE, Schlusselberg DS, Jeffery R, Hayden S, Cromwell LD, Pluta D, English RA: Three-dimensional computed tomographic angiography in the preoperative evaluation of cerebrovascular lesions. Neurosurgery 36: 320-326, 1995

11) Harrison MJ, Johnson BA, Gardner GM, Welling BG: Preliminary results on the management of unruptured intracranial aneurysms with magnetic resonance angiography and computed tomographic angiography. Neurosurgery 40: 947-955, 1997

12) Hashimoto $H$, Iida J, Hironaka $Y$, Okada M, Sakaki T: Use of spiral computerized tomography angiography in patients with subarachnoid hemorrhage in whom subtraction angiography did not reveal cerebral aneurysms. J Neurosurg 92: 278-283, 2000 
13) Heiserman JE, Dean BL, Hodak JA, Flom RA, Bird CR, Drayer BP, Fram EK:. Neurologic complications of cerebral angiography. AJNR Am J Neuroradiol 15: 1401-1407, 1994

14) Hoh BL, Cheung AC, Rabinov JD, Pryor JC, Carter BS, Ogilvy CS: Results of a prospective protocol of computed tomographic angiography in place of catheter angiography as the only diagnostic and pretreatment planning study for cerebral aneurysms by a combined neurovascular team. Neurosurgery 54 : 1329-1340, 2004

15) Hope JK, Wilson JL, Thomson FJ: Three-dimensional CT angiography in the detection and characterization of intracranial berry aneurysms. AJNR Am J Neuroradiol 17: 439-445, 1996

16) Hsiang JN, Liang EY, Lam JM, Zhu XL, Poon WS: The role of computed tomographic angiography in the diagnosis of intracranial aneurysms and emergent aneurysm clipping. Neurosurgery 38: 481-487, 1996

17) Katz DA, Marks MP, Napel SA, Bracci PM, Roberts SL: Circle of Willis: evaluation with spiral CT angiography, MR angiography, and conventional angiography. Radiology 195: 445-449, 1995

18) Koenig GH, Marshall WH Jr, Poole GJ, Kramer RA: Rupture of intracranial aneurysms during cerebral angiography: report of ten cases and review of the literature. Neurosurgery 5: 314-324, 1979

19) Komiyama M, Tamura K, Nagata Y, Fu Y, Yagura H, Yasui T: Aneurysmal rupture during angiography. Neurosurgery 33: 798-803, 1993

20) Korogi Y, Takahashi M, Katada K, Ogura Y, Hasuo K, Ochi M, Utsunomiya H, Abe T, Imakita S: Intracranial aneurysms: detection with three-dimensional CT angiography with volume rendering-comparison with conventional angiographic and surgical findings. Radiology 211: 497-506, 1999

21) Kuhn J, Vehlen C, Mennel HD, Mahkorn D, Bewermeyer $\mathrm{H}$ : Rupture of an internal carotid artery aneurysm during angiography with leakage of contrast medium via an external ventricular drain. Neuroradiology 45: 905-907, 2003

22) Lai PH, Yang CF, Pan HB, Chen C, Ho JT, Hsu SS: Detection and assessment of circle of Willis aneurysms in acute subarachnoid hemorrhage with three-dimensional computed tomographic angiography: correlation with digital subtraction angiography findings. J Formos Med Assoc 98: 672-677, 1999

23) Liang EY, Chan M, Hsiang JH, Walkden SB, Poon WS, Lam WW, Letreweli C: Detection and assessment of intracranial aneurysms: value of CT angiography with shaded-surface display. AJR Am J Roentgenol 165: 1497-1502, 1995

24) Matsumoto $M$, Sato $M$, Nakano $M$, Endo $Y$, Watanabe Y, Sasaki T, Suzuki K, Kodama N: Threedimensional computerized tomography angiographyguided surgery of acutely ruptured cerebral aneurysms. J Neurosurg 94: 718-727, 2001
25) Nakajima $Y$, Yoshimine T, Yoshida H, Sakashita K, Okamoto M, Kishikawa M, Yogi K, Yokota J, Hayakawa T: Computerized tomography angiography of ruptured cerebral aneurysms: factors affecting time to maximum contrast concentration. J Neurosurg 88: 663-669, 1998

26) Ogawa T, Okudera T, Noguchi K, Sasaki N, Inugami A, Uemura K, Yasui N: Cerebral aneurysms: evaluation with three-dimensional CT angiography. AJNR Am J Neuroradiol 17: 447-454, 1996

27) Pechlivanis I, Schmieder K, Scholz M, Konig M, Heuser L, Harders A: 3-Dimensional computed tomographic angiography for use of surgery planning in patients with intracranial aneurysms. Acta Neurochir (Wien) 147: 1045-1053, 2005

28) Saitoh H, Hayakawa K, Nishimura K, Okuno Y, Teraura T, Yumitori K, Okumura A: Rerupture of cerebral aneurysms during angiography. AJNR Am J Neuroradiol 16: 539-542, 1995

29) Seruga T, Bunc G, Klein GE: Helical high-resolution volume-rendered 3-dimensional computer tomography angiography in the detection of intracranial aneurysms. J Neuroimaging 11: 280-286, 2001

30) Strayle-Batra M, Skalej M, Wakhloo AK, Ernemann U, Klier R, Voigt K: Three-dimensional spiral CT angiography in the detection of cerebral aneurysm. Acta Radiol 39: 233-238, 1998

31) Tanabe S, Ohtaki M, Uede T, Hashi K, Suzuki S, Takahashi H: [Diagnosis of ruptured and unruptured cerebral aneurysms with three-dimensional CT angiography (3D-CTA)]. No Shinkei Geka 23: 787-795, 1995 (Japanese)

32) Velthuis BK, Rinkel GJ, Ramos LM, Witkamp TD, Berkelbach van der Sprenkel JW, Vandertop WP, van Leeuwen MS: Subarachnoid hemorrhage: aneurysm detection and preoperative evaluation with CT angiography. Radiology 208: 423-430, 1998

33) Velthuis BK, Van Leeuwen MS, Witkamp TD, Ramos LM, Berkelbach van Der Sprenkel JW, Rinkel GJ: Computerized tomography angiography in patients with subarachnoid hemorrhage: from aneurysm detection to treatment without conventional angiography. J Neurosurg 91: 761-767, 1999

34) Villablanca JP, Hooshi P, Martin N, Jahan R, Duckwiler G, Lim S, Frazee J, Gobin YP, Sayre J, Bentson J, Vinuela F: Three-dimensional helical computerized tomography angiography in the diagnosis, characterization, and management of middle cerebral artery aneurysms: comparison with conventional angiography and intraoperative findings. J Neurosurg 97: 1322-1332, 2002

35) Waugh JR, Sacharias N: Arteriographic complications in the DSA era. Radiology 182: 243-246, 1992

36) White PM, Teasdale EM, Wardlaw JM, Easton V: Intracranial aneurysms: CT angiography and MR angiography for detection prospective blinded comparison in a large patient cohort. Radiology 219: 739-749, 2001

37) White PM, Wardlaw JM Easton V: Can noninvasive 
imaging accurately depict intracranial aneurysms? A systematic review. Radiology 217: 361-370, 2000

38) Wintermark M, Uske A, Chalaron M, Regli L, Maeder P, Meuli R, Schnyder P, Binaghi S: Multislice computerized tomography angiography in the evaluation of intracranial aneurysms: a comparison with intraarterial digital subtraction angiography. J Neurosurg 98: 828-836, 2003

39) Young N, Dorsch NW, Kingston RJ, Markson G, McMahon J: Intracranial aneurysms: evaluation in 200 patients with spiral CT angiography. Eur Radiol 11: 123-130, 2001

40) Zouaoui A, Sahel M, Marro B, Clemenceau S, Dargent N, Bitar A, Faillot T, Capelle L, Marsault C: Three-dimensional computed tomographic angiography in detection of cerebral aneurysms in acute subarachnoid hemorrhage. Neurosurgery 41: 125-130, 1997

Address reprint requests to: Mutsumi Nagai, M.D., Department of Neurosurgery, Jichi Medical University, 3311-1 Yakushiji, Shimotsuke, Tochigi 329-0498, Japan.

e-mail:mnagai@jichi.ac.jp

\section{Commentary}

The authors achieved excellent diagnostic and surgical outcomes for intracranial aneurysms using only 3D CT angiography with a diagnostic sensitivity up to $100 \%$. They also describe valuable tips for creating $3 \mathrm{D}$ presurgical evaluation images of cerebral aneurysms. These could shorten mean preoperative time delays up to 2.8 hours, reducing rebleeding rates considerably. Since preoperative time-delay and intra-arterial digital subtraction angiography (DSA) might be risk factors for aneurysmal rebleeding, shortening such delays via 3D CT angiography offers definite practical benefits over conventional catheter-based cerebral angiography in reducing preoperative rebleeding and avoiding complications from this invasive procedure. In addition, the $3 D$ reconstruction produces a useful operative view for preoperative surgical planning, giving anatomical information about the aneurysm itself and the adjacent neurovascular and bony structures. Since the introduction of multichannel 3D CT angiography, surgeons have the possibility of fast and accurate vascular image acquisition. Such angiographies have replaced DSA in some institutes, especially in cases of ruptured aneurysms with large hematomas. However, 3D CT angiography is limited in its ability to aid evaluations of collateral circulation, venous drainage, and hemodynamic information of blood flow. DSA is still the gold standard diagnostic tool for intracranial aneurysms. There are several advantages in using only $3 \mathrm{D} C \mathrm{CT}$ angiography for aneurysm surgery, but if there are any diagnostic difficulties or need for further presurgical information, surgeons should not hesitate to order DSA.

Dae Hee HAN, M.D., Ph.D. Department of Neurosurgery

Director

Cerebral and Cardiovascular Disease Center National Medical Center Seoul, R.O.K. 